\title{
Experiences with Distributed Heterogeneous Clouds over Community Networks
}

\author{
Mennan Selimi, Felix Freitag, \\ Daniel Marti \\ Universitat Politècnica de Catalunya \\ Barcelona, Spain \\ \{mselimi, felix\}@ac.upc.edu \\ \{daniel.marti.i\}@est.fib.upc.edu
}

\author{
Roger Pueyo Centelles, Pau Escrich, \\ Roger Baig \\ Fundació Privada per a la Xarxa Lliure, \\ Oberta i Neural guifi.net \\ Barcelona, Spain \\ \{roger.pueyo, pau.escrich, \\ roger.baig\}@guifi.net
}

\begin{abstract}
Introduction: Community networks [1] are decentralized and self-organized communication networks built and operated by citizens and for citizens. They are an emergent model of infrastructure that aims to satisfy a community's demand for Internet and ICT services. There are several large community networks in Europe having from 500 to 20000 nodes, such as Guifi.net ${ }^{1}, \mathrm{AWMN}^{2}$, FunkFeuer ${ }^{3}$ and many more worldwide. Most of them are based on Wi-Fi technology, but also a growing number of optical fiber links start to become deployed.

Problem statement: Cloud-enabled services in community networks have not yet been demonstrated. Most users see in community networks only the possibility to gain Internet access. Sharing of the bandwidth between nodes enables the traffic from users to be routed over the nodes of different node owners. Since this is done in a reciprocal manner, community networks successfully operate as IP networks. However, the sharing of other computing resources like storage, which is now common practice in today's Internet through cloud computing, hardly exists in community networks, making community networks lose an important opportunity for offering a new set of innovative services that could be based on collective actions. Different to the general purpose cloud-based applications offered in the Internet, clouds in community networks [2] would 1) allow providing cloud-based services that are relevant for the community, and 2) create services that are shaped and owned by the community.
\end{abstract}

Our approach: Our approach is to show the feasibility of such community clouds by an experimental assessment of cloud-based applications running on real clouds within

\footnotetext{
${ }^{1}$ http://guifi.net

${ }^{2}$ http://awmn.gr

${ }^{3}$ http://funkfeuer.at
}

the Guifi community network. We experiment with two distributed file systems deployed over a heterogeneous distributed cloud infrastructure. Tahoe-LAFS and XtreemFS run on distributed cloud resources provided by KVM-based VMs from Proxmox and OpenStack cloud management platforms, on Linux containers (LXC) from a community resource management platform and even on storage space allocated on IoT embedded boards. The distributed file systems for the experiment were selected according their potential relevance for community network users, covering storage solutions for different requirements regarding fault tolerance, storage performance and privacy. We evaluate the performance of Tahoe-LAFS and XtreemFS in this highly diverse setting and in the context of the dynamic conditions of the community network. In the experiments the write and read throughput of Tahoe-LAFS and XtreemFS are measured. Each experiment is repeated several times and with different file sizes to assess the storage systems.

Contribution: The contribution of this paper is an experimental study on the feasibility and performance of two distributed file systems deployed on clouds in community networks. We observed that both file system performed functionally correct. Tahoe-LAFS by design offers end-toend encryption and fault-tolerance to churn of nodes. As such it seems to be particularly suitable as a solution for storage use cases in community networks such as personal backups where privacy of data is important.

Our findings should stimulate further application deployments, the uptake of clouds by community networks, and encourage the network and systems research community to help solving important research challenges of community network clouds related to self-management and scalability, to make protocols, services and applications more resilient and adaptive.

\section{REFERENCES}

[1] B. Braem et al. A case for research with and on community networks. SIGCOMM Comput. Commun. Rev., 43(3):68-73, July 2013.

[2] J. Jimenez et al. Supporting Cloud Deployment in the Guifi.net Community Network. In 5th Global Information Infrastructure and Networking Symposium (GIIS 2013), Trento, Italy, Oct. 2013.
Permission to make digital or hard copies of part or all of this work for personal or classroom use is granted without fee provided that copies are not made or distributed for profit or commercial advantage and that copies bear this notice and the full citation on the first page. Copyrights for third-party components of this work must be honored. For all other uses, contact the Owner/Author. Copyright is held by the owner/author(s). DCC'14, August 18, 2014, Chicago, Illinois, USA. Copyright 2014 ACM 978-1-4503-2992-7/14/08. http://dx.doi.org/10.1145/2627566.2627581. 